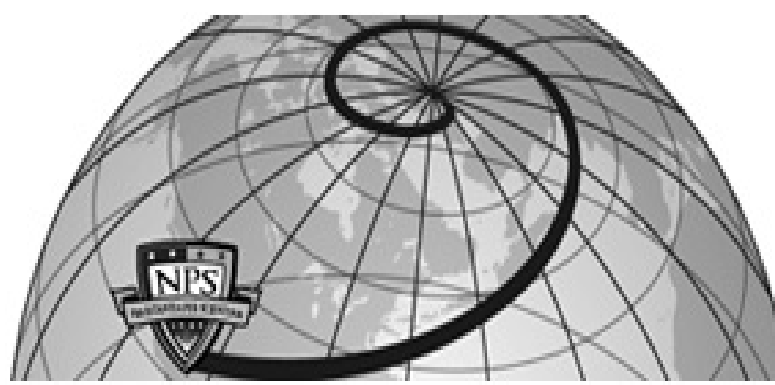

Calhoun: The NPS Institutional Archive DSpace Repository

\title{
Radiation produced by the modulated electron beam of a free electron laser
}

Neighbours, John Robert; Maruyama, Xavier K.; Buskirk, Fred Ramon

Monterey, California. Naval Postgraduate School

https://hdl.handle.net/10945/29059

This publication is a work of the U.S. Government as defined in Title 17, United States Code, Section 101. Copyright protection is not available for this work in the United States.

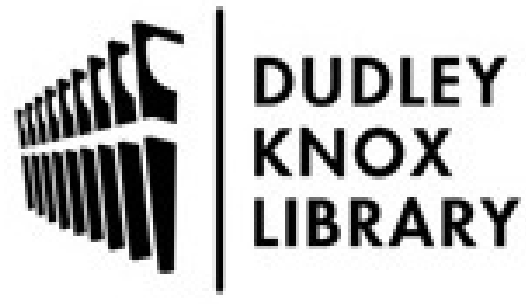

http://www.nps.edu/library
Calhoun is the Naval Postgraduate School's public access digital repository for research materials and institutional publications created by the NPS community. Calhoun is named for Professor of Mathematics Guy K. Calhoun, NPS's first appointed -- and published -- scholarly author.

Dudley Knox Library / Naval Postgraduate School 411 Dyer Road / 1 University Circle Monterey, California USA 93943 


\title{
NAVAL POSTGRADUATE SCHOOL. Monterey, California
}

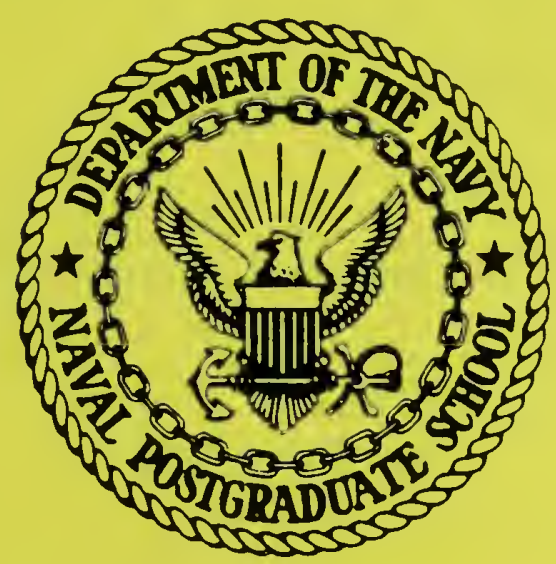

\section{RADIATION PRODUCED BY THE MODULATED ELECTRON BEAM OF A FREE ELECTRON LASER}

By

\author{
Fred R. Buskirk, John R. Neighbours \\ and \\ Xavier K. Maruyama \\ June 1986 \\ Technical Report
}

FEDDOCS

D 208.14/2:

NPS-61-86-013

Approved for public release; distribution unlimited

cepared for: Defense Advanced Research Projects Agency Arlington, VA 2209-2308 
NAVAL POSTGRADUATE SCHOOL

Monterey, California

Rear Admiral R. H. Shumaker

Superintendent

D. A. Schrady

Provost

The work reported herein was supported in by Defense Advanced Research Project Agency.

Reproduction of all or part of this report is authorized.

This report was prepared by: 


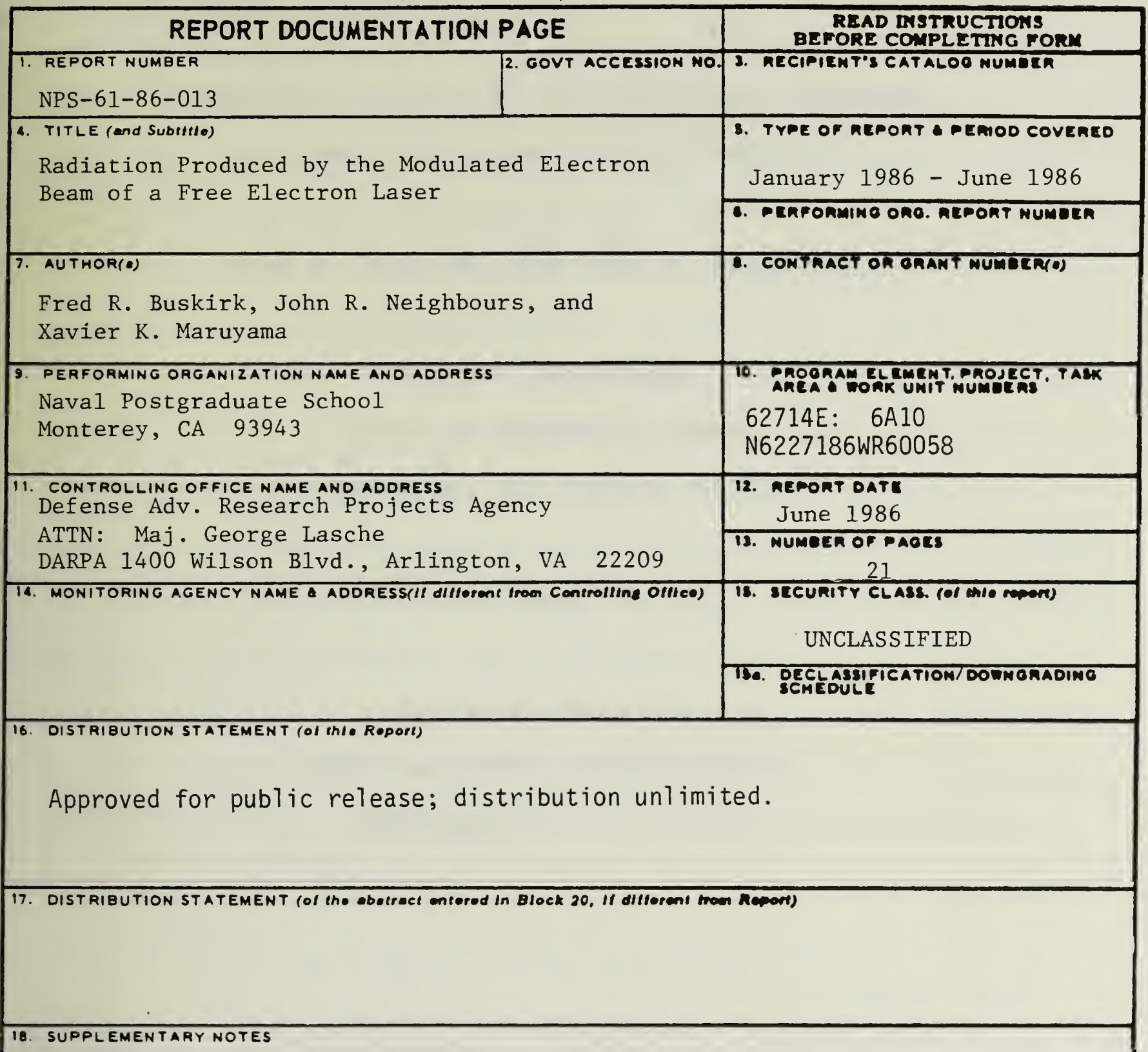

9. KEY WORDS (Continue on reveree elde II neceeeary and Identlty by block number)

Electron beams, Free Electron Lasers, Cerenkov Radiation

\section{ABSTAACT (Continue on reveree olde II neceseant and ldonlity by Olock mabber)}

The electron beam in a free electron laser (FEL) becomes axially modulated at the optical wave length of the FEL radiation. This electron beam passed through a gas may produce intense Cerenkov radiation. The effects of the radial and axial dimension of the electron bunches on the radiation are explored. 

Radiation Produced by the Modulated Electron

Beam of a Free Electron Laser

Fred R. Buskirk, and John R. Neighbours

Physics Department

Naval Postgraduate School

Monterey, California 93943

and

Xavier K. Maruyama

National Bureau of Standards

Gaithersburg, MD 20899

\section{ABSTRACT}

The electron beam in a free electron laser (FEL) becomes axially modulated at the optical wave length of the FEL radiation. This electron beam passed through a gas may produce intense Cerenkov radiation. The effects of the radial and axial dimension of the electron bunches on the radiation are explored. 


\section{Background}

In several publications, 1,2 we have explored the coherent Cerenkov radiation produced by electron beam bunches in a dielectric medium, such as air. The radiation from a single electron is weak, yielding of the order of ten optical photons per meter in air. The beam bunches from an $r . f$ accelerator, with $10^{11}$ electrons per bunch, radiate coherently at low frequencies which allows radiation in the microwave range. The factor of $10^{4}$ loss, occurring because the intensity is preportional to the frequency, is more than offset by the increase resulting from coherence. The coherent radiation tends to cancel at higher frequencies, in which the wavelength of the radiation is smaller than bunch size. The size is about $1 \mathrm{~cm}$ for the bunch from an $\mathrm{S}$-band accelerator implying a maximum radiated frequency of about $30 \mathrm{G} \mathrm{Hz}$.

\section{Basic Formulation}

The electron beam from a free electron laser (FEL) offers the possibility of observing and using coherent cerenkov radiation at optical frequencies and their relatively high output power. The electron beam in the FEL, after interacting with the wiggler and radiation fields, must become modulated in the axial direction. This "wasted" electron beam, usually stiff because it is relativistic, could maintain its modulation, pass into a gas cell and produce coherent Cerenkov radiation. The resulting radiation could be used to diagnose the electron beam modulation. At higher currents the radiation intensity might be a significant 
power source, although, breakdown of the gas would be a limitation. Hollow dielectric wave guides, successful for the microwaves 5 , have not been used in the optical range, but could possibly be used to avoid problems associated with passing the beam directly through the dielectric medium.

III. Cerenkov Radiation from Charges and Charge Distributions Frank and Tamm $^{6}$ deduced the power radiated by a charge moving at velocity $v$ in a medium in which the radiation velocity is $c$, and $v>c$. The radiation velocity in free space is $c_{0}$ and we let $\cos \theta_{c}=c / v$, where $c=(\mu \varepsilon)^{-1 / 2}$ and $c_{0}$ is the free space value, the radiation is produced in a cone propagating at an angle $\theta_{c}$ with respect to $v$. The radiation has a continuous spectrum, and in the frequency range dw, the radiated power for an infinite path length is

where

$$
P(\omega) d \omega=\frac{\mu V}{4 \pi} q^{2} \sin ^{2} \theta_{c} \omega d \omega F^{2}(\vec{k})
$$

$$
F(\vec{k})=q^{-1} \iiint e^{i \vec{k} \cdot \vec{r}} \rho_{o}(\vec{r}) d v,
$$

and $\rho_{0}(\vec{r})$ is the charge density evaluated in the lab coordinate system at $t=0$. The vector $\vec{k}$ is in the direction of the emitted radiation and $k=\omega / c . \quad F(\vec{k})$ may be called the form factor of the charge distribution, and it is normalized to be unity in the limit $\vec{k}=0$. For a point charge $F(\vec{k})$ is identically one for all. values of $\vec{k}$. The actual forms of Eq. 1 and 2 are those given in Ref. 1,2 and reduce to the Frank and Tamm results if $F=1$. 
For a periodic series of charge bunches, the corresponding derivation predicts radiation appearing at harmonics $w$ of the bunch angular frequency $\omega_{0}$, with the power given by

$$
P(\omega)=\frac{\mu v}{4 \pi} \quad \omega \omega_{0} \sin ^{2} \theta_{c} F^{2}(\vec{k}) q^{2}
$$

for an infinite path length. Here $q$ and $P(\omega)$ are the charge and the power radiated per bunch. $F(\vec{k})$ is still defined by Eq.2, and in this periodic case, the integration is carried out over a single bunch. Eq.3 is derived by completely classical reasoning and has been confirmed 1,7 for radiation in the microwave range for bunch sizes about $1 \mathrm{~cm}$ in length and emitted radiation up to $30 \mathrm{GHz}$. In the optical range where quantum effects might be expected, Eq.1 is satisfied for point charges (with $F=1$ ). We assume that the Eq. 3 holds in the optical range. The charge distribution must be specified to proceed. Let $s^{2}=x^{2}+y^{2}$ and

$$
\rho_{O}(\vec{r})=\rho_{r}(\vec{s}) \rho_{L}(z)
$$

With this form for $\rho_{O}(r)$, the form factor becomes

$$
F(\vec{k})=F_{r}\left(\vec{k}_{S}\right) F_{z}\left(k_{z}\right)
$$

The charge per bunch, $q$ is

$$
q=I \ell / v
$$


where $I$ is the current, $\ell$ is the spacing between bunches, $v$ is the bunch speed.

Letting $\mathrm{N}$ be the harmonic number, the power radiated per bunch is

$$
P(\omega)=\pi \mu N \vee \sin ^{2} \theta_{c} I^{2} F^{2}
$$

which depends on the current in the bunch, not on the total charge. Also, the radiation is more efficient for large $N$ if the form factor $F$ allows such high harmonics. Finally, the efficiency increases with $I$, because the beam power is proportional to I but the radiated power varies as $I^{2}$. To consider the efficiency for radiation further, Eq. 7 may be manipulated. The energy radiated per unit length by one bunch is $P / v$, while the energy required to form the bunch is $q V$ or $I \ell V / v$, where $V$ is the accelerating voltage in Volts. If the radiating medium is of length $L$, we may form the dimensionless ratio $E($ radiated)/E(beam) which from Eq. 6 become

$$
E(\mathrm{rad}) / E(\text { beam })=\pi N Z_{\circ} \frac{I}{V} \frac{L}{l} \cos \theta_{c} \sin ^{2} \theta_{c} F^{2}
$$

where $Z_{0}$ is the impedence of the medium, i.e., $Z_{0}=(\mu / \varepsilon)^{1 / 2}$. Let $I=10^{2} \mathrm{~A}, \mathrm{~V}=10^{8}$ Volts, $\sin \theta_{\mathrm{C}}=10^{-2}, \mathrm{~F}=1, \mathrm{~L}=1 \mathrm{~m}$ and $\ell=10^{-6} \mathrm{~m}$. Then $E(\mathrm{rad}) / E($ beam $)$ is about $10 \%$. Thus fairly high efficiency for 
radiation may be achieved for possible values of $\mathrm{L} / \ell$, and extremely high efficiency could occur if $\ell$, the beam period, is in the optical region, as realized in a free electron laser. The main problem is the small value of $F$ which results for a wide beam.

IV. Specific Considerations for an FEL Beam.

The Equations developed $(\mathrm{Eq} \cdot 7,8)$ describe the radiation by a periodic electron beam. The main problem comes from the form factor $F$ which describes the charge distribution. For an FEL, the axial charge distribution is modulated at the optical wave length, of the order of microns, but the radial beam dimensions may be as large as millimeters. Then $F$, which describes diffraction, may be very small. To proceed, we consider a modulated beam, in which $\rho_{L}(z)$ in $E q .4$ is given by a cosine function, which restricts harmonic production to $N=1$. Thus,

$$
\rho_{L}(z)=1+A \cos (K z)
$$

We may let $\rho_{r}(s)$ be axially symmetric, in the form of a uniform disc of radius $r$, or a gaussian in the form of $\exp \left(-s^{2} / a^{2}\right)$. For either case, Eq.9 yields

$$
F_{z}\left(k_{z}\right)=\delta_{k_{z}, 0}+\frac{A}{2} \delta_{k_{z}, K}+\frac{A}{2} \delta_{k_{z},-K}
$$

From the general considerations of Ref.1,2, the vector $\vec{k}$ is at an angle $\theta_{C}$ to the beam axis, the radiation at any frequency 
stays in phase with the electron bunch, and $k_{S}=k \sin \theta_{C}$. From Eq.10 with $k_{z}=K$, the radiation matches the periodicity of the electron beam, and higher harmonics in Eq.9 would give $k_{z}=N K$. Note that because $\omega=c k$ for the radiation and $\omega_{0}=v K$ for the beam, and $k_{z}=k \cos \theta_{C}$ we have, setting $k_{z}=N K$, cos $\theta_{c}=c / v$ gives $\omega=N w_{0}$. This means that the Cerenkov radiation, in the medium with velocity $c$, has the same frequency as the FEL radiation in vacuum, or an integer multiple thereof.

For the disc of radius $r$,

$$
F_{r}\left(k_{s}\right)=2 J_{1}\left(k_{s} r\right) / k_{s} r
$$

For the gaussion radial distribution,

$$
F_{r}\left(k_{s}\right)=e^{-k_{s}^{2}} a^{2} / 4
$$

Both form factors arise from a non-zero radial distribution of charge, and both yield a great suppression of radiation from diffraction from a finite source when the radius parameter ( $r$ or a) is many times larger than the wave length of the radiation. If the beam radius parameters in Eq.11 and 12 are $10^{-3} \mathrm{~m}$, the radiation has $\lambda=10^{-6} \mathrm{~m}$ and $\theta_{C}=1.3^{\circ}$ for air, and is very small for the gaussian, because in $\mathrm{F}_{\mathrm{r}}{ }^{2}=-5.09 \times 10^{3}$. For the disc, $\mathrm{k}_{\mathrm{s}} \mathrm{r}$ $=141.5$ and $F_{r}{ }^{2}=8.79 \times 10^{-7}$ if the envelope of the oscillating function of Eq.11 is considered. 
To conclude this section, we substitute the results of Eq.9, 10 into Eq.7 to obtain the power radiated per (optical) bunch of the electron beam

$$
P(\omega)=\frac{\pi \mu}{4} N v \sin \theta_{C} I^{2} A^{2} F_{r}^{2}\left(\vec{k}_{S}\right)
$$

In this form the parameter A describes the axial modulation of the charge according to Eq.9. If harmonics other than $N=1$ are considered, Eq. 9 becomes a Fourier series and depends on $\mathrm{N}$. V. Enhancement of FEL Cerenkov Radiation

The previous section shows that the FEL electron beam, because of its short axial modulation length is potentially an efficient radiator but a wide radial dimension may severely limit the radiation, by diffraction. Eq.11, 12 are identical to optical diffraction by uniform and gaussian shaded apertures, but the Cerenkov condition requires the vector $\vec{k}$ to be on the Cerenkov cone, which picks out a narrow ring far out on the circular diffraction pattern. The radiation could be increased by at least three methods (a) concentration of the same current into a smaller radius, (b) passing the beam through an aperture, which would decrease the current but increase the form factor, and (c) bending the beam in a magnet. The last two are considered in more detail below

A. Radiation Enhancement - Aperture

Let the beam pass through an aperture of small radius $r^{\prime}$ The gaussian beam then becomes a uniform disc with a form factor 
given by Eq.11 with $r^{\prime}$ replacing $r$. Using the envelope form $2 J_{1}(x) / x \rightarrow\left(8 / \pi x^{3}\right) 1 / 2$,

$$
\frac{{ }_{A} A}{P}=\frac{8}{\pi\left(k_{s} r^{\prime}\right)^{3}}{\frac{\left(r^{\prime}\right)^{4}}{a^{4}}}^{+}+k_{s}^{2} a^{2} / 4
$$

where $P A$ and $P$ are the powers with and without the aperture. The power increase may be very large, but only because the gaussian originally caused strong suppression.

For the disc beam, the result is

$$
\frac{P A}{P}=\frac{r^{-}}{r}
$$

There is a loss of power for the disc, because the square of the form factor is proportional to $\left(r^{-}\right)^{-3}$ but $q^{2}$ varies as $\left(r^{-}\right)^{4}$

B. Radiation Enhancement-Deflected Beam

Let the beam velocity be deflected by an angle $\theta_{c}$ by $a$ magnet. The planes of the charge discs will be unchanged so that the normal to the disc will be at an angle $\theta_{c}$ to the velocity. Part of the Cerenkov cone will then be perpendicular to the disc with no diffraction. Let $\phi$ be the azimuth angle of $\vec{k}$ in the Cerenkov cone, relative to the direction in which $\vec{k}$ is perpendicular to the disc. As the direction of $\vec{k}$ changes, the first diffraction null occurs for

$$
\frac{|\Delta \vec{k}|}{|\vec{k}|}=1.22 \frac{\lambda}{2 r}
$$


The fraction $f$ of the Cerenkov cone within this first diffraction lobe is then approximately

$$
f=\left|\frac{\Delta \vec{k}}{2 \pi k}\right|_{S}=\frac{1.22}{2 \pi} \quad \frac{\lambda}{2 r} \quad \frac{1}{\sin \theta_{C}}
$$

For the given $\lambda, r$ and $\theta_{c}, f=4.28 \times 10^{-3}$.

The effect is that this small fraction $f$ of the cone

produces radiation, which is relatively strong, with $\mathrm{Fr}_{\mathrm{r}^{2}}=1$ in this region, as opposed to $\mathrm{Fr}^{2}=8.79 \times 10^{-7}$ for all the cone of the undeflected beam. Thus the power output is enhanced greatly over the diffraction-suppressed radiation without the magnetic deflection.

VI. Summary

The modulated beam from an FEL will produce Cerenkov radiation if it passes through air, if the energy is above threshold. This radiation is potentially very strong, because the electron bunches radiate coherently and the efficiency increases with frequency. Realistic beams pose a problem because the relatively large radial dimensions result in diffractive suppression of the radiation. In the text, two methods are considered to alleviate the diffraction loss-passing the beam through an aperature, and bending it in a magnetic field. The latter method seems more promising for the assumed beam parameters. 
These calculations are based on classical electrodynamics. The results are verified in the usual point particle Cerenkov effect in the optical range, and coherent emission by beam bunches in the microwave range. But here, a new process is explored-coherent Cerenkov emission but in the optical range. Experiments should be done, to explore the physics in this range, as well as provide diagnostics for FEL beams. 


\section{REFERENCES}

1. F. R. Buskirk and J. R. Neighbours, Phys. Rev. A28, 1531 (1983)

2. J. R. Neighbours and F. R. Buskirk, Phys. Rev. A29, 3246 (1984)

3. F. R. Buskirk and J. R. Neighbours, Phys. Rev. A31, 3750 (1985)

4. F. R. Buskirk and J. R. Neighbours, Phys. Rev. (To be published)

5. M. Danos, Journ. Appl. Phys. 26, 2 (1955)

6. I. Tamm, J. Phys. SSSR 1, 439 (1939)

7. X. K. Maruyama, J. R. Neighbours, F. R. Buskirk, D. D. Snyder, M. Vujaklija and R. G. Bruce, Journ. Appl. Phys. (To be published) 
Figure 1. The modulated bunches produce Cerenkov radiation, but a relatively large radius produces diffraction losses.

Figure 2. The charge bunches are bent in a magnetic field, which changes the direction of $\vec{v}$ but leaves the plane of the disc unchanged.

Figure 3. The ray shown by $\vec{k}$ is on the Cerenkov cone and normal to the disc, so radiation is strong. This strength will persist out to an angle $\theta$ such that diffraction produces a null. Thus part of the Cerenkov cone produces strong radiation. 

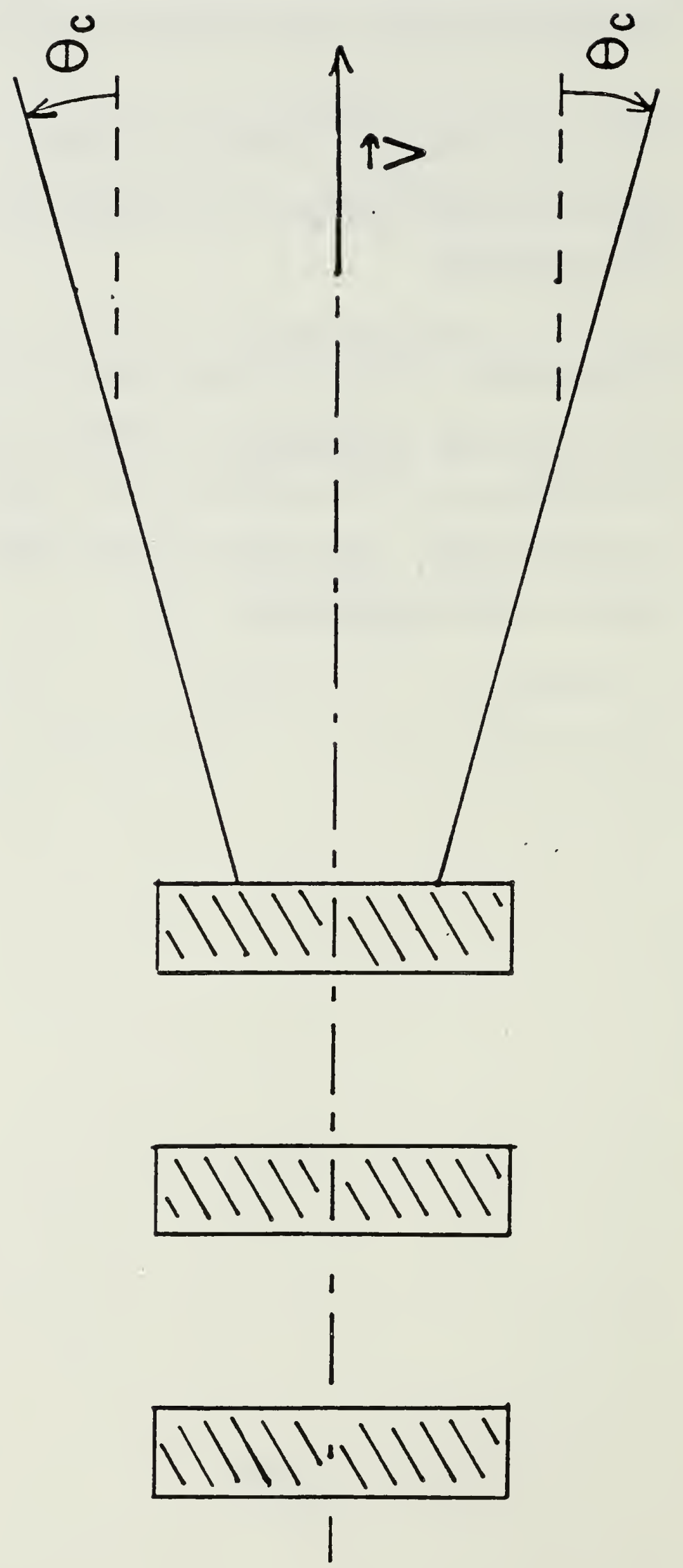

$-14-$ 


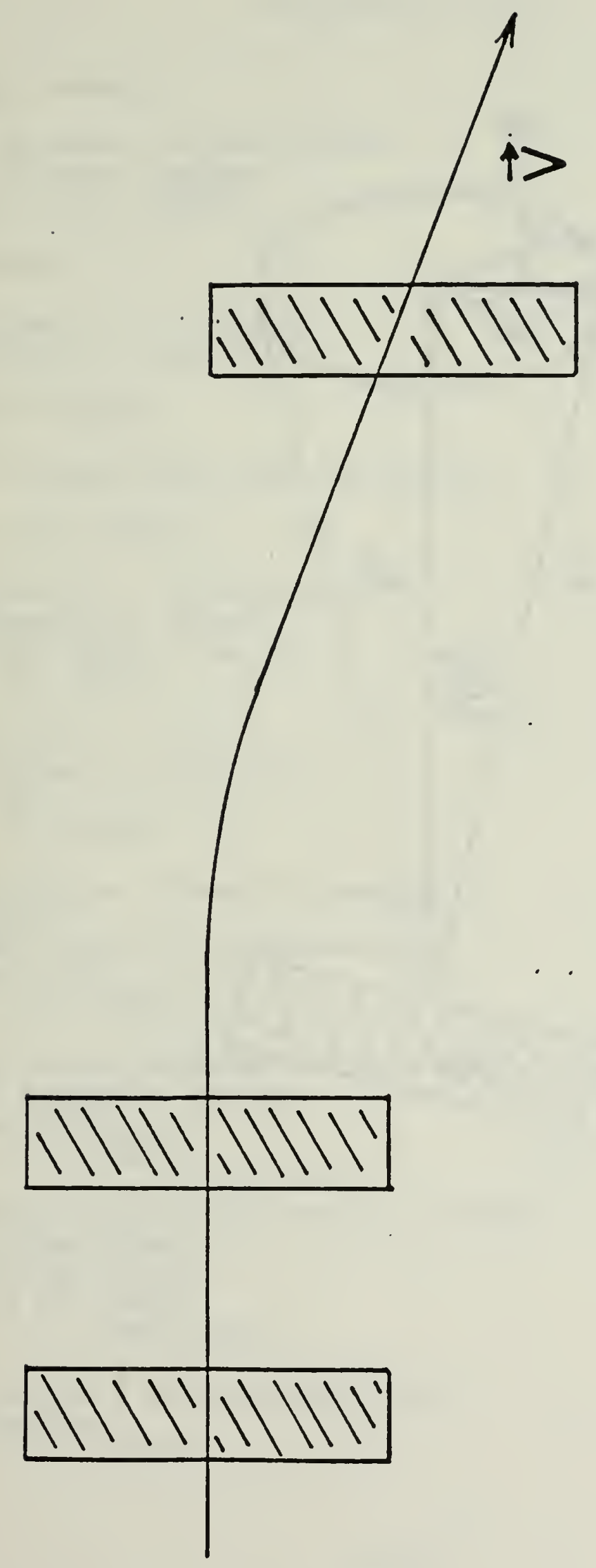




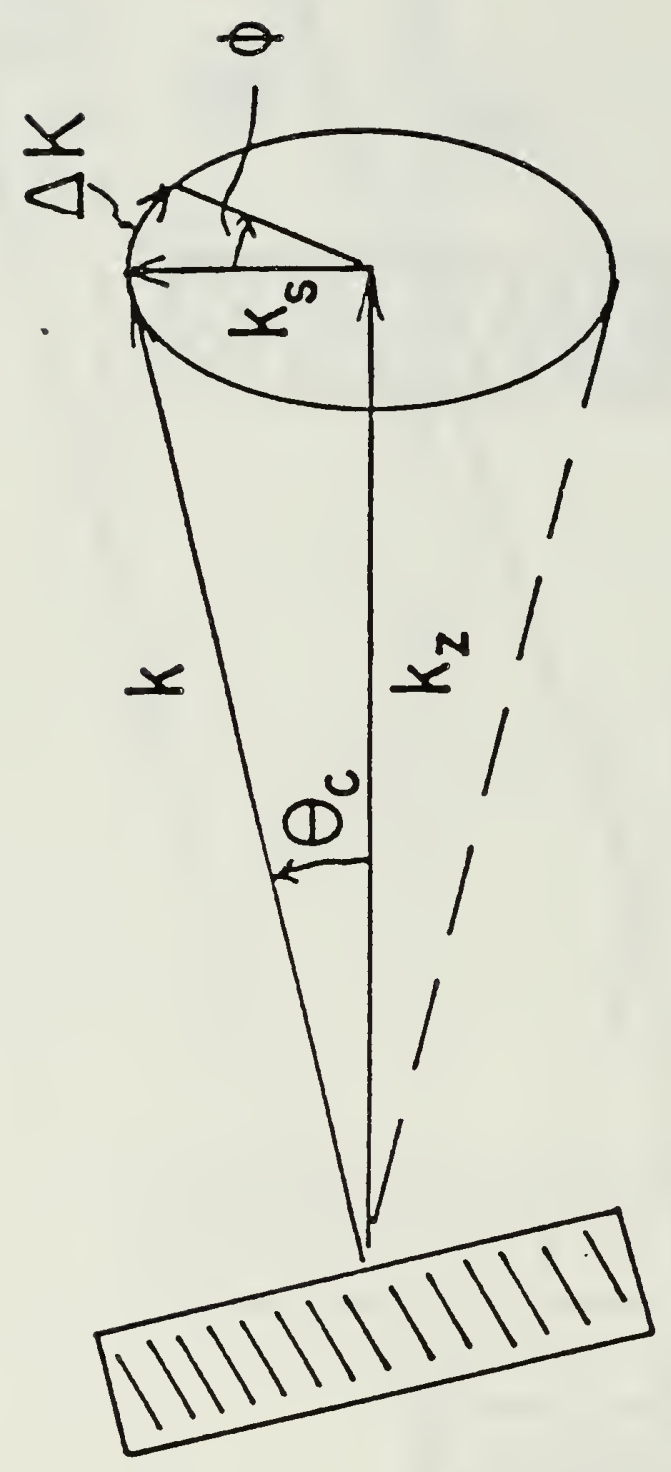


CDR William Bassett

PMS 405

Strategic Systems Project Office

Naval Sea Systems Command

Washington, D.C. 20376

Dr. H. Boehmer

TRW

One Space Park

Redondo Beach, CA 92078

Dr. Richard Briggs

$L-321$

Lawrence Livermore National Laboratory

Box 808

Livermore, CA 94550

F. R. Buskirk \& J. R. Neighbours

Naval Postgraduate School

Physics Department, Code 61

Monterey, CA 93943

Dr. W. Colson

Berkeley Research Associates

P. 0. Box 241

Berkeley, CA 94701

Director, Defense Advanced Research

Project Agency

ATTN: LCOL Richard A. Gullickson

1400 Wilson Blvd.

Arlington, CA 22209

Defense Advanced Research Project Agency

ATTN: MAJ George P. Lasche

1400 Wilson Blvd.

Arlington, VA 22209

Defense Advanced Research Projects Agency

Attn: Dr. Shen Shey

Directed Energy office

1400 Wilson Boulevard

Arlington, VA 22209-2308

Defense Technical Information Center 
Physics Department

UCSB

Santa Barbara, CA 93106

Dr. K. Felch

Varian Corporation

611 Hansen Way

Palo Alto, CA 94303

Dr. V. L. Granatstein

Electrical Engineering Dept.

University of Maryland

College Park, MD 20742

Dr. C. M. Huddleston

ORI, Inc.

1375 Piccard Drive

Rockville, MD 20850

La Jolla Institute

Attn: Dr. K. Brueckner

P. O. Box 1434

La Jolla, CA 92038

Lawrence Berkeley Laboratory

Attn: Dr. Edward P. Lee

Building 47, Room 111

1 Cycltron Road

Berkeley, CA 94720

Lawrence Livermore National Laboratory

Univeristy of California

Attn: Dr. William A. Barletta

Dr. Daniel S. Prono

Dr. Adrian C. Smith

Dr. Simon S. YU

Dr. John T. Weir

Dr. Thomas J. Karr

Dr. William M. Fawley

Dr. Eugene J. Lauer

Dr. George J. Caporaso

P. O. BOX 808

Ms. Lois Barber

Livermore, CA 94550

Library

Code 0142

Naval Postgraduate School

Monterey, CA 93943 
Dr. Joseph Mack

M4, M.S. P-940

Los Alamos National Laboratory

Los Alamos, NM 87545

Dr. J. Madey

Department of Physics

Stanford University

Stanford, CA 94305

Prof. T. C. Marshall

Dept. of Applied Physics and Nuclear Engineering

Columbia University

New York, NY 10027

Dr. Xavier K. Maruyama

Bldg. 245, Room R-108

National Bureau of Standards

Gaithersburg, MD 20899

Dr. David Merritt

PMS 405

Strategic Systems Project Office

Naval Sea Systems Command

Washington, D.C. 20376

Mission Research Corporation

Attn: Dr. N. J. Carron

P. O. Box 719

Santa Barbara, CA 93102

Mission Research Corporation

Attn: Dr. Brendan B. Godfrey

Dr. Larry Wright

Dr. Barry Newberger

Dr. R. Adler

Dr. G. Kiuttu

Dr. T. Hughes

Dr. Dushan Mitrovitch

Plasma Sciences Division

1720 Randolph Road, SE

Albuquerque, NM 87106 
Naval Surface Weapons Center

White Oak Laboratory

Attn: Dr. Eugene E. Nolting (R401)

Dr. Andy Smith (H23)

Ms. Beverly McLean (R401)

Dr. H. C. Chen (R41)

Dr. Han S. Uhm (R41)

Dr. Ralph Fiorito (R41)

Dr. John Smith (R41)

Dr. Donald Rule (R41)

Dr. M. J. Rhee (R41)

10901 New Hampshire Avenue

Silver Springs, MD 20903-5000

Office of Naval Research

CDR James offutt

1030 East Green Street

Pasadena, CA 91106

Office of Naval Research

CDR R. Swafford

800 N. Quincy Street

Arlington, VA 22217

Office of Research Administration

Code 012

Naval Postgraduate School

Monterey, CA 93943

Dr. C. Pellegrini

Brookhaven National Laboratory

Bldg 902

Accelerator Dept.

Upton, NY 11973

CAPT Kurt Stevens

Patrick AFB

Patrick, FL 32925

Strategic Defense Initiative Organization

Directed Energy Weapons Office

The Pentagon

Attn: LTCOL Richard L. Gullickson

Office of the Secretary of Defense

Washington, D.C. 20301-7100

Dr. Kenneth W. Struve

Lawrence Livermore National Laboratory

P. 0. Box 808

Livermore, CA 94550 
Admiral R. L. Topping

Space and Naval Warfare Systems Command

SPAWAR-06

Washington, D.C. 20363-5100

LCDR E. Turner

PMS 405

Strategic Systems Project office

Naval Sea Systems Command

Washington, D.C. 20376

Dr. R. Warren

Los Alamos Scientific Laboratory

P. O. Box 1663

Los Alamos, NM 87545 



$$
\text { U228341 }
$$


